\title{
Femoral and popliteal venous Doppler during prone and supine position in COVID-19 patients: a potential diagnostic tool to detect abnormal right ventricular function
}

\author{
Alexandra Malinovska, MD · Ketina Arslani, MD • Núria Zellweger • \\ Catherine Gebhard, MD, PhD · William Beaubien-Souligny, MD · Alexander Calderone, MD • \\ Martin Siegemund, MD - Markus Aschwanden, MD - André Denault, MD, \\ PhD $\cdot$ Caroline E. Gebhard, MD
}

Received: 16 November 2020/Revised: 28 December 2020/Accepted: 29 December 2020/Published online: 2 February 2021

(C) Canadian Anesthesiologists' Society 2021

\section{To the Editor,}

Acute respiratory distress syndrome (ARDS) is a frequent complication of severe coronavirus disease (COVID-19). Acute respiratory distress syndrome increases right ventricular (RV) afterload and is a known etiology of RV dysfunction. Right ventricular dilatation and dysfunction have been linked to higher mortality in patients with COVID-19. ${ }^{1}$ Therefore, careful observation of RV function is important to guide supportive therapy. In COVID-19 pneumonia, the prevalence of ARDS is high and the prone position (PP) is often used, making noninvasive hemodynamic monitoring more challenging with limited access to the echocardiographic windows for up to 16 hrs. Nevertheless, alternative assessment tools such as

Supplementary Information The online version of this article (https://doi.org/10.1007/s12630-021-01918-2) contains supplementary material, which is available to authorized users.

\footnotetext{
A. Malinovska, MD · N. Zellweger - C. E. Gebhard, MD (ه) . Department of Intensive Care Medicine, University Hospital Basel, Basel, Switzerland

e-mail: evacaroline.gebhard@usb.ch

K. Arslani, MD

Department of Cardiology, University Hospital Basel, Basel, Switzerland

C. Gebhard, $\mathrm{MD}, \mathrm{PhD}$

Center for Molecular Cardiology, University of Zurich, Zurich, Switzerland

Department of Nuclear Medicine, University Hospital Zurich, Zurich, Switzerland

W. Beaubien-Souligny, MD

Nephrology Division, Department of Medicine, Centre

Hospitalier de l'Université de Montréal, Montreal, QC, Canada
}

Doppler interrogation of the femoral vein to diagnose right heart dysfunction have recently been described and correlated to abnormal pulse-wave Doppler profiles of the portal and renal venous circulation. ${ }^{2}$ In the PP, the popliteal vein becomes perfectly exposed and offers an indirect sonographic window to the heart. The use of femoral and popliteal venous Doppler interrogation to infer RV function has not yet been reported in COVID-19 patients with ARDS.

Under physiologic conditions, veins with normal compliance are collapsible and pressure waves arising from the cardiac cycle are dampened in the periphery. Normal peripheral vein Doppler waveforms are mostly continuous, unidirectional, and have a signal that is independent from the cardiac cycle, but are associated with respiratory changes (i.e., respiratory modulation). A consensus statement has recently been released regarding

\author{
A. Calderone, MD \\ Department of Anesthesiology, Université de Montréal, \\ Montreal, QC, Canada \\ M. Siegemund, MD \\ Department of Intensive Care Medicine, University Hospital \\ Basel, Basel, Switzerland \\ Department of Clinical Research, University of Basel, Basel, \\ Switzerland \\ M. Aschwanden, MD \\ Department of Angiology, University Hospital Basel, Basel, \\ Switzerland
}

\author{
A. Denault, $\mathrm{MD}, \mathrm{PhD}$ \\ Department of Anesthesiology and Critical Care Division, \\ Montreal Heart Institute, Montreal, QC, Canada
}



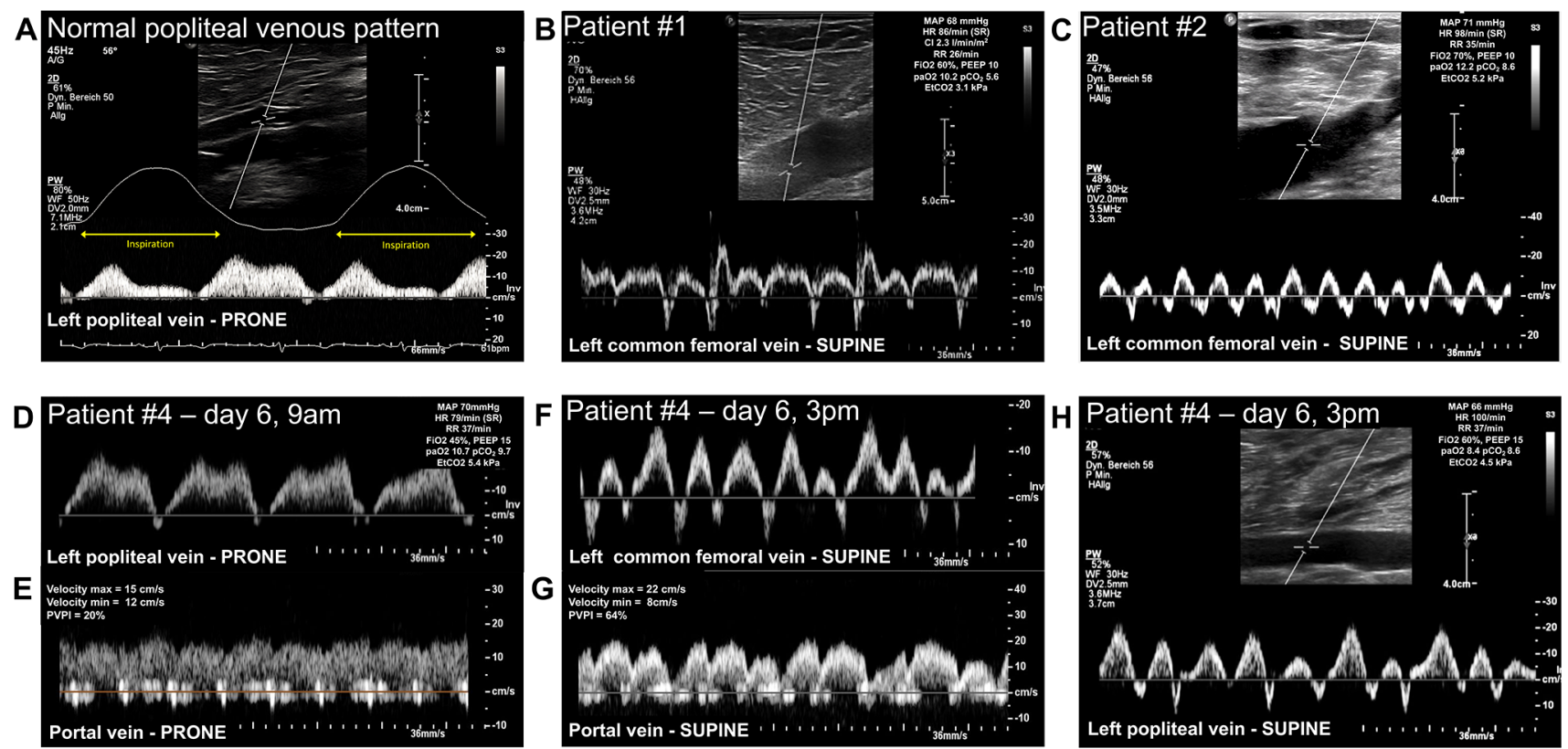

Figure A) example of a normal popliteal vein Doppler pattern during prone position (PP) with only antegrade velocities. Note the correlation with the electrocardiogram and respiratory variation. Echocardiography (only apical views) at the same time revealed normal right ventricular (RV) function. B) 58-yr old male (patient \#1) with central pulmonary embolism and acute RV failure and multiorgan dysfunction. B-mode and spectral Doppler profile of the left common femoral vein in supine position shows absence of respiratory variation and a bidirectional velocity profile with a significant retrograde component $\left(>10 \mathrm{~cm} \cdot \mathrm{sec}^{-1}\right)$. C) 70-yr old woman (patient \#2) with cor pulmonale from long standing ARDS. Spectral Doppler profile of the left common femoral vein in supine position. Note the abnormal, pulsatile signal without respiratory modulation. Echocardiography showed RV dilatation and an estimated systolic pulmonary artery pressure of $62 \mathrm{mmHg}$ using echocardiography and central venous pressure of $17 \mathrm{mmHg}$. D) 65-yr old male (patient \#4) with COVID-19 pneumonia complicated by severe ARDS. Duplex ultrasound on day 6 in PP showed only slightly abnormal Doppler profile of the popliteal vein: note the only mild retrograde velocities (retrograde velocities $<$ $5 \mathrm{~cm} \cdot \mathrm{sec}^{-1}$ ) along with absence of respiratory modulation. E) Accordingly, a normal spectral Doppler profile of the portal vein was observed with a portal venous pulsatility index (PVPI) of $20 \%$ (PI = [maximal-minimal velocity $] /$ mean velocity). Six hours later back in supine position, the Doppler profile differed clearly, now showing a strong pulsatile velocity pattern in the F femoral vein, G portal vein (PVPI 64\%) and $\mathbf{H}$ popliteal vein, suspicious of $\mathrm{RV}$ dysfunction. $\mathrm{CI}=$ cardiac index; $\mathrm{EtCO}_{2}=$ end-expiratory carbon dioxide; $\mathrm{F}_{1} \mathrm{O}_{2}=$ fraction of inspired oxygen; $\mathrm{HR}=$ heart rate $\mathrm{MAP}=$ mean arterial pressure; $\mathrm{paO}_{2}=$ arterial partial pressure of oxygen; $\mathrm{paCO}_{2}=$ arterial partial pressure of carbon dioxide; $\mathrm{PEEP}=$ positive end-expiratory pressure; PVPI = portal venous pulsatility index; RR = respiratory rate

the definition of abnormal peripheral venous Doppler signal. $^{3}$ Right ventricular dysfunction associated with elevated right atrial pressure causes abnormal pulse-wave transmission through the distended veins and can be imaged in every peripheral venous vessel accessible to Doppler interrogation. In patients with RV dysfunction, an abnormal femoral or popliteal vein Doppler is characterized by synchrony with the cardiac cycle (cardiac pulsatile modulation) with a phasic or bidirectional pulsatile wave form as the right atrial pressure increases. A pulsatile femoral vein on Doppler interrogation is highly specific $(89-100 \%)$ for elevated right atrial pressure. ${ }^{4}$

We assessed 21 critically ill patients admitted to the intensive care unit with COVID-19-related ARDS to detect deep vein thrombosis and to identify abnormal femoral or popliteal venous Doppler signals. All patients or substitute decision makers provided written consent for the presentation of their data. In five patients, an abnormal femoral or popliteal venous Doppler pattern was detected. In two patients, popliteal venous Doppler was performed during PP. These observations were consistent with echocardiographic findings in the supine position, confirming the suspicion of RV dysfunction. Panel A in the Figure depicts a normal popliteal vein Doppler profile during PP, while panels B-H show abnormal femoral or popliteal venous Doppler tracings of mechanically ventilated patients in the prone or supine position. In panels E,G of the Figure, the corresponding pulse-wave Doppler of the portal vein is shown. For all five patients, demographic, clinical, and laboratory data are provided in the Electronic Supplementary Material data (eTable).

While transthoracic echocardiographic assessment of $\mathrm{RV}$ function is challenging in the PP, the transesophageal approach was recently described to track the hemodynamic response of the PP in COVID-19 patients with ARDS. ${ }^{5}$ Nevertheless, Doppler interrogation of the femoral or popliteal vein — the latter easily exposed in the PP—may be 
simpler and faster with a lower exposure risk for the operator and less invasive for the patient. Of course, the trade-off is that less actionable information is obtained.

This technique may be of special interest when time is scarce or advanced cardiac ultrasound techniques are not readily available. Currently, Doppler interrogation of the femoral or popliteal vein may serve as a screening tool to heighten the suspicion of right heart dysfunction even when performed by physicians inexperienced in ultrasound. Our observations will need further investigation and, ultimately, validation in a prospective setting.

Disclosures André Denault: CAE-Healthcare (2010) speaker, (2020) consultant; Masimo (2017) speaker, (2020) consultant; Edwards (2019) speaker and research (equipment grant) all unrelated with this current report. CG has received speakers' fees from Sanofi Genzyme, travel support from Siemens Healthineers, and research support from the Novartis Foundation and Bayer Pharmaceuticals. The Department of Nuclear Medicine, University Hospital Zurich, holds a research contract with GE Healthcare. All are unrelated with this current report. The other authors have no conflicts of interest to declare.

Funding statement This study was supported by the Swiss National Science Foundation grant\# 31CA30196140 and the LOOP Zurich, to CEG, CG and MS. CEG was supported by grants from the Research Foundation in Anesthesiology and Intensive Care Medicine, University Hospital Basel and the Research Foundation of the University of Basel. AD is supported by the Richard I. Kaufman Endowment Fund in Anesthesia and Critical Care and the Montreal Heart Institute Foundation. KA has received a research grant from the
Swiss Academy of Medical Sciences and the Bangerter-RhynerFoundation, Switzerland (\#YTCR 09/19).

Editorial responsibility This submission was handled by Dr. Philip M. Jones, Deputy Editor-in-Chief, Canadian Journal of Anesthesia.

\section{References}

1. Li Y, Li H, Zhu S, et al. Prognostic value of right ventricular longitudinal strain in patients with COVID-19. JACC Cardiovasc Imaging 2020; 13: 2287-99.

2. Denault AY, Aldred MP, Hammoud A, et al. Doppler interrogation of the femoral vein in the critically ill patient: the fastest potential acoustic window to diagnose right ventricular dysfunction? Crit Care Explor 2020; DOI: https://doi.org/10.1097/CCE. 0000000000000209.

3. Kim ES, Sharma AM, Scissons R, et al. Interpretation of peripheral arterial and venous Doppler waveforms: a consensus statement from the Society for Vascular Medicine and Society for Vascular Ultrasound. Vasc Med 2020; 25: 484-506.

4. Alimoglu E, Erden A, Gursel K, Olcer T. Correlation of right atrial pressure and blood flow velocities in the common femoral vein obtained by duplex Doppler sonography. J Clin Ultrasound 2001; 29: 87-91.

5. Evrard B, Goudelin M, Fedou AL, Vignon P. Hemodynamic response to prone ventilation in COVID-19 patients assessed with 3D transesophageal echocardiography. Intensive Care Med 2020; 46: 2099-101.

Publisher's Note Springer Nature remains neutral with regard to jurisdictional claims in published maps and institutional affiliations. 